\title{
An Analytical Model of Microsegregation in Alloy Solidification
}

\author{
Y. H. SHIN, M. S. KIM, K. S. OH, ${ }^{1)}$ E. P. YOON ${ }^{21}$ and C. P. HONG \\ Center for Computer-Aided Materials Processing (CAMP), Department of Metallurgical Engineering Yonsei University, Seoul, \\ Korea. $\quad$ 1) Iron and Steel Making Research Group, Posco Technical Research Lab., Pohang, Korea. \\ 2) Hanyang University. E-mail: hong@yonsei.ac.kr.
}

(Received on August 28, 2000; accepted in final form on October 26, 2000)

\begin{abstract}
An analytical model has been developed for modeling microsegregation in the secondary arm spacings of binary alloys. The present model includes the dilution effects both of back-diffusion and coarsening on microsegregation of solute in solidification of binary alloys. When the dilution effect of coarsening is not considered, the present model approaches to the Kobayashi model, whilst it approaches to the Mortensen model in the absence of back-diffusion. The present model was verified by the comparison with other previous analytical models, and successfully applied to the prediction of eutectic fractions in directional solidification of an $\mathrm{Al}-4.9 \mathrm{wt} \% \mathrm{Cu}$ alloy. The predicted eutectic fractions by the present model are in good agreement with the measured ones. It was found that the present microsegregation model is one of the most appropriate analytical models to account for both the effects of back-diffusion and coarsening.
\end{abstract}

KEY WORDS: microsegregation; secondary dendrite arms; solidification; coarsening; back-diffusion; dilution; analytical solution.

\section{Introduction}

In dendritic solidification of alloys, the solute rejected at the solid/liquid interface is redistributed at the scale of the secondary dendrite arm spacings, resulting in the formation of microsegregation, which affects the composition of the microstructure and the fraction of eutectic or other phases. Several analytical models of microsegregation have been reported and recently reviewed by Kraft and Chang. ${ }^{1)}$ There are two key features which affect the solute balance and redistribution in dendritic solidification. One is the back-diffusion of solute into the solid and the other the coarsening of the microstructure. It is well known that both the backdiffusion and the coarsening dilute the solute in the liquid, and affect the amount of eutectic or other phases. ${ }^{2}$

The analytical models of microsegregation can be classified into three groups. The first type of microsegregation model suggested by Brody-Flemings, ${ }^{3)}$ Clyne-Kurz, $\left.{ }^{4}\right)$ Ohnaka, ${ }^{5)}$ and Kobayashi ${ }^{6}$ accounts for back-diffusion but neglecting coarsening. The second type of model accounting for coarsening but neglecting back-diffusion was presented by Mortensen, ${ }^{7)}$ in the case of a constant cooling rate. Recently, The third type, recently proposed by Voller and Beckermann, ${ }^{8,9)}$ accounts for both back-diffusion and coarsening. However, the model still has some limitations since the effect of coarsening on the dilution of the liquid phase was indirectly accounted for by adding a dimensionless diffusion parameter to the back-diffusion Fourier number.

In the present study, an analytical model of microsegregation, in which both the effects of back-diffusion and coarsening are accounted for, has been developed. The present model was compared with other analytical models and the experimental results on eutectic fractions in directional solidification of an $\mathrm{Al}-4.9 \mathrm{wt} \% \mathrm{Cu}$ alloy reported in the literature. $^{10)}$

\section{Analytical Model}

\subsection{Basic Assumptions}

To establish a microsegregation model, a representative volume element in the two-phase mushy region in a solidifying alloy is defined to be the half-arm spacing in a platelike morphology shown in Fig. 1. In order to derive the governing equations for this problem, the following assumptions are used.

(i) The analysis domain is one-dimensional as shown in Fig. 1 and is specified by the coordinate.

$$
0 \leq X(t) \leq L(t)
$$

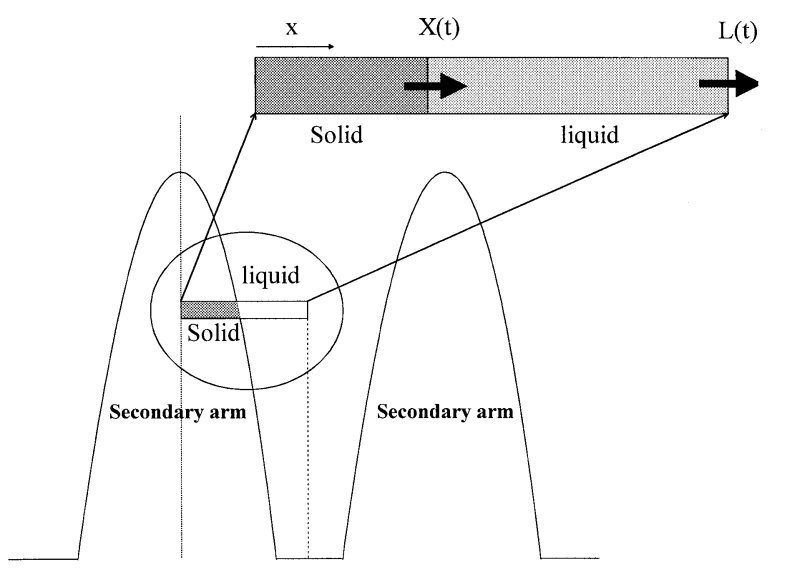

Fig. 1. A schematic diagram of the calculation domain. 
where $X(t)$ is the position of the solidification front and $L(t)$ is a free surface located at the midpoint of the secondary arm spacing which is continuously expanding by introducing new liquid as the secondary dendrite arm coarsens. Solidification is assumed to be controlled by the following scheme.

$$
X(t)=L_{\mathrm{f}}\left(\frac{t}{t_{\mathrm{f}}}\right)^{1 / m}
$$

where $m$ indicates the reciprocal growth exponent; 1 for a linear growth and 2 for a parabolic growth. The calculation domain length $L(t)$, which indicates the coarsening of secondary dendrite arms, is assumed to be given by

$$
L(t)=L_{\mathrm{f}}\left(\frac{t}{t_{\mathrm{f}}}\right)^{1 / n}
$$

where $t$ indicates time and $t_{\mathrm{f}}$ the local solidification time. $L_{\mathrm{f}}\left(=\lambda_{2} / 2\right)$ is the final length of the calculation domain defined by the half-spacing at the completion of solidification. $\lambda_{2}$ represents the secondary dendrite arm spacing. $n$ is the reciprocal coarsening exponent.

(ii) Thermophysical properties such as density, diffusivity and partition coefficient are assumed to be constant.

(iii) Mass transfer in the calculation domain is controlled by diffusion alone. Perfect mixing of solute in liquid is assumed. The solute balance in a representative volume element is preserved by

$$
\int_{0}^{X} C_{\mathrm{S}}(x, t) d x+[L(t)-X(t)] C_{\mathrm{L}}(t)=L(t) C_{0}
$$

where $C_{\mathrm{S}}$ and $C_{\mathrm{L}}$ indicate the concentrations of solid and liquid, and $C_{0}$ is the initial concentration. In order to solve Eq. (4), the following boundary and initial conditions are used.

$$
\begin{aligned}
& \frac{\partial C_{\mathrm{S}}}{\partial x}=0 \quad \text { at } x=0 \ldots \ldots \\
& C_{\mathrm{L}}=C_{0} \quad \text { at } t=0 \quad(X=0)
\end{aligned}
$$

(iv) The concentration profile in the solid domain is assumed to be given by the Ohnaka's approximation ${ }^{5)}$

$$
C_{\mathrm{S}}(x, t)=\mathrm{C}_{\mathrm{S}}^{*}(t)+a\left[x^{2}-X^{2}(t)\right]
$$

Here the parameter $a$ is considered to be a function of time, and $C_{\mathrm{S}}^{*}$ represents the solid concentration on the solidification front.

\subsection{Governing Equations}

By differentiating Eq. (4) by $t$ and applying the boundary condition (5), we have

$$
C_{\mathrm{L}}(1-k) \frac{d X}{d t}=\left.D_{\mathrm{S}} \frac{\partial C_{\mathrm{S}}}{\partial x}\right|_{x=X}+\frac{d C_{\mathrm{L}}}{d t}(L-X)+\left(C_{\mathrm{L}}+C_{0}\right) \frac{d L}{d t}
$$

(C)

where $k$ is the equilibrium partition coefficient. The terms (A) through (D) in the above equation are schematically shown in Fig. 2. The term (A) indicates the amount of

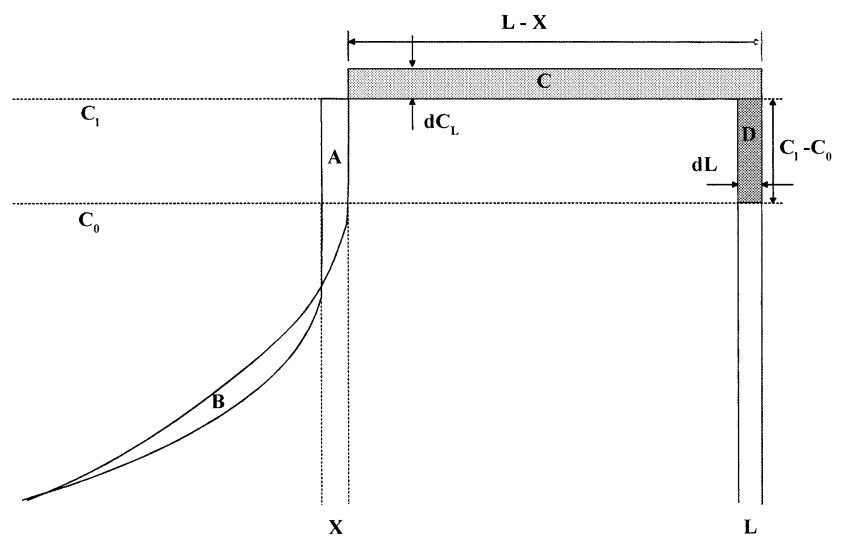

Fig. 2. Solute conservation with considering back diffusion and coarsening.

Table 1. Dimensionless variables and relationships.

\begin{tabular}{c|l}
\hline Dimensionless variables & $t^{\prime}=\frac{t}{t_{f}}, L^{\prime}=\frac{L}{L_{f}}, X^{\prime}=\frac{X}{L_{f}}, C_{L}^{\prime}=\frac{C_{L}}{C_{0}}$ \\
\hline & $t^{\prime}=\left(X^{\prime}\right)^{m}, L^{\prime}=\left(t^{\prime}\right)^{\frac{1}{n}}, L^{\prime}=\left(X^{\prime}\right)^{\frac{m}{n}}$, \\
$\begin{array}{c}\text { Relationships between the } \\
\text { dimensionless variables }\end{array}$ & $\frac{d t^{\prime}}{d X^{\prime}}=m\left(X^{\prime}\right)^{m-1}, \frac{d L^{\prime}}{d X^{\prime}}=\frac{m}{n}\left(X^{\prime}\right)^{\frac{m-n}{n}}$ \\
\hline
\end{tabular}

solute rejected into the liquid at the solidification front, (B) the back-diffusion term, (C) the increase of solute in the liquid, and (D) the coarsening term.

The back-diffusion term of Eq. (8) is a partial derivative. It was simplified to an ordinary derivative by substituting Eq. (7) into Eq. (8) as follows.

$$
\frac{d C_{\mathrm{L}}}{d t}(L-X)=C_{\mathrm{L}}(1-k) \frac{d X}{d t}-2 a D_{\mathrm{S}} X-\left(C_{\mathrm{L}}-C_{0}\right) \frac{d L}{d t}
$$

By applying the dimensionless variables defined in Table 1 into Eq. (9), we obtain

$$
\frac{d C_{\mathrm{L}}^{\prime}}{d t}\left(L^{\prime}-X^{\prime}\right)=C_{\mathrm{L}}^{\prime}(1-k) \frac{d X^{\prime}}{d t^{\prime}}-\frac{2 a t_{\mathrm{f}} D_{\mathrm{S}} X^{\prime}}{C_{0}}-\left(C_{\mathrm{L}}^{\prime}-1\right) \frac{d L^{\prime}}{d t^{\prime}}
$$

From Eqs. (4) and (7), $a$ is given by

$$
\begin{aligned}
a & =\frac{3}{2} \frac{\left(C_{\mathrm{L}}-C_{0}\right) L+C_{\mathrm{L}}(k-1) X}{X^{3}} \\
& =\frac{3}{2} \frac{C_{0}}{L_{\mathrm{f}}^{2}} \frac{\left(C_{\mathrm{L}}^{\prime}-1\right) L^{\prime}+C_{\mathrm{L}}^{\prime}(k-1) X^{\prime}}{X^{\prime 3}}
\end{aligned}
$$

By substituting Eq. (11) into Eq. (10) and multiplying both sides of Eq. (10) by $d t^{\prime} / d X^{\prime}$, we have the following equation.

$$
\begin{aligned}
& \frac{d C_{\mathrm{L}}^{\prime}}{d X^{\prime}}\left(L^{\prime}-X^{\prime}\right)=C_{\mathrm{L}}^{\prime}(1-k) \\
& -3 \alpha \frac{\left(C_{\mathrm{L}}^{\prime}-1\right) L^{\prime}+C_{\mathrm{L}}^{\prime}(k-1) X^{\prime}}{X^{\prime 2}} \frac{d t^{\prime}}{d X^{\prime}}-\left(C_{\mathrm{L}}^{\prime}-1\right) \frac{d L^{\prime}}{d X^{\prime}}
\end{aligned}
$$

where $\alpha\left(\equiv D_{\mathrm{S}} t_{\mathrm{f}} / L_{\mathrm{f}}^{2}\right)$ is the Fourier number. Equation (12), 
Table 2. Classification of analytical models for microsegregation.

\begin{tabular}{|c|c|c|c|}
\hline Coarsening & $D_{S} \rightarrow \infty$ & $D_{S}=$ finite & $D_{S}=0$ \\
\hline No coarsening & $\begin{array}{c}\text { Equilibrium } \\
\text { (lever rule) }\end{array}$ & $\begin{array}{c}\text { Brody-Flemings model } \\
\text { Clyne-Kruz model } \\
\text { Ohnaka model } \\
\text { Kobayashi model }\end{array}$ & Scheil \\
\hline Coarsening & $\begin{array}{c}\text { Voller-Beckerniain model } \\
\text { Presert madel }\end{array}$ & Mortensen model \\
\hline
\end{tabular}

which is the governing equation for solute transport in a coarsening microstructure, can be simply expressed as an ordinary differential equation of $X^{\prime}$ by applying the relationships given in Table 1.

$$
\frac{d C_{\mathrm{L}}^{\prime}}{d X^{\prime}}=A C_{\mathrm{L}}^{\prime}+B
$$

where $A$ and $B$ are defined as follows:

$$
\begin{aligned}
A= & \left.\left(X^{\prime}\right)^{\frac{m}{n}}-X^{\prime}\right)^{-1} \\
& \times\left[1-k-3 \alpha m\left(X^{\prime}\right)^{m-2}\left(\left(X^{\prime}\right)^{\frac{m-n}{n}}+k-1\right)-\frac{m}{n}\left(X^{\prime}\right)^{\frac{m-n}{n}}\right] \\
& B=m\left(\left(X^{\prime}\right)^{\frac{m}{n}}-X^{\prime}\right)^{-1}\left[3 \alpha\left(X^{\prime}\right)^{\frac{m+m n-3 n}{n}}+\frac{1}{n}\left(X^{\prime}\right)^{\frac{m-n}{n}}\right]
\end{aligned}
$$

The combined effects of coarsening and back-diffusion are included in the present model, given by Eqs. (13)-(15). The dimensionless solute concentration of liquid, $C_{\mathrm{L}}^{\prime}\left(X^{\prime}\right)$, is determined by four parameters such as the partition coefficient $k$, the reciprocal growth exponent $m$, the reciprocal coarsening exponent $n$, and the Fourier number $\alpha$.

\subsection{Solution Scheme}

Equation (13) is an ordinary differential equation which has the complexity in getting an algebraic solution. Thus, the 4th order Runge-Gutta method was adopted to calculate the concentration of liquid. From the ordinary differential equation and the initial condition, the concentration of liquid can be obtained. The concentration of solid at the solid/liquid interface can also be calculated using the equilibrium partition coefficient.

\section{Results and Discussion}

As described in Table 2, microsegregation models are classified into three groups: the first one includes the effect of back-diffusion only, the second one the effect of coarsening only, and the third one both the effects of back-diffusion and coarsening.

\subsection{Limiting Cases of the Present Model}

As described in the previous section, the combined effects of coarsening and back-diffusion are included in the present model, given by Eqs. (13)-(15). In order to verify the present model, two limiting cases will be examined as follows.
(1) Back-diffusion in the Absence of Coarsening

When the effect of coarsening is not considered, $n$ becomes infinite. Then, Eqs. (14) and (15) can be simplified to

$$
\begin{aligned}
& A=\left(1-X^{\prime}\right)^{-1}\left[1-k-3 \alpha m\left(X^{\prime}\right)^{m-2}\left(\left(X^{\prime}\right)^{-1}+k-1\right)\right] \ldots \\
& B=m\left(1-X^{\prime}\right)^{-1}\left[3 \alpha\left(X^{\prime}\right)^{m-3}\right] \text {. }
\end{aligned}
$$

In this case, most of the basic assumptions used in the present model are similar to those in the Ohnaka's model. ${ }^{5}$ ) However, the solution of the present model is different from that of Ohnaka's model since the definition of the parameter $a$ is not identical to each other. In the present model the parameter $a$ is given by Eq. (11) as a function of $X(t)$, while it was considered as a constant in the Ohnaka's model. It is thus considered that the present model is more reasonable to account for the back diffusion than the Ohnaka's model.

Figure 3 compares the computational results of the present model with other analytical models in the absence of coarsening. Fig. 3(a) shows that the present model shows a better agreement with the second order approximation of the Kobayashi model than other models in case of $\alpha=$ 0.237 . The Kobayashi approximation has been known as the most reasonable solution to account for the effect of back-diffusion. ${ }^{6}$ ) As the value of $\alpha$ which accounts for the effect of back-diffusion decreases, all computational results become similar, as shown in Figs. 3(b) and 3(c), and finally approach to that of Scheil model.

(2) Coarsening in the Absence of Back-diffusion

When the effect of back-diffusion is not considered, $\alpha$ becomes zero. Then, Eqs. (14) and (15) can be rewritten as

$$
\begin{gathered}
A=\left(\left(X^{\prime}\right)^{\frac{m}{n}}-X^{\prime}\right)^{-1}\left[1-k-\frac{m}{n}\left(X^{\prime}\right)^{\frac{m-n}{n}}\right] \ldots \ldots . . \\
B=m\left(\left(X^{\prime}\right)^{\frac{m}{n}}-X^{\prime}\right)^{-1}\left[\frac{1}{n}\left(X^{\prime}\right)^{\frac{m-n}{n}}\right] \ldots \ldots \ldots \ldots
\end{gathered}
$$

Equation (13) can also be solved with the simplified parameters given by Eqs. (18) and (19).

Mortensen has proposed a microsegregation model under a constant cooling rate, in which the effect of coarsening is accounted for but the effect of back-diffusion is not considered as follows. ${ }^{7)}$

$$
C_{\mathrm{L}}=C_{0}+\beta t \text {. }
$$

Here $\beta=-R / m_{\mathrm{L}}, R$ is a constant cooling rate, and $m_{\mathrm{L}}$ is the liquidus slope. The final solution of the Mortensen model is given by the following equation.

$$
f_{\mathrm{S}}=\frac{1+\frac{1}{n}}{1-k} \frac{\left(C_{\mathrm{L}}^{\prime}\right)^{\frac{1}{k-1}}}{\left(C_{\mathrm{L}}^{\prime}-1\right)^{\frac{1}{n}}} \int_{1}^{C_{\mathrm{L}}^{\prime}}\left(C^{\prime}\right) \frac{k}{1-k}\left(C^{\prime}-1\right)^{\frac{1}{n}} d C^{\prime}
$$

where $f_{\mathrm{S}}$ indicates the solid fraction defined by

$$
f_{\mathrm{S}}(t)=\frac{X(t)}{L(t)}
$$

However, the final form of the Mortensen model is derived under an assumption of a constant cooling rate. It is there- 


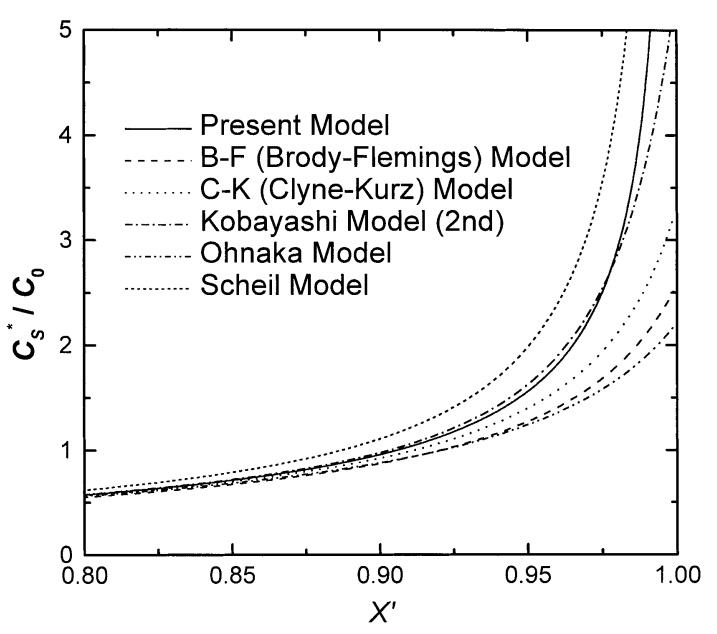

(a)

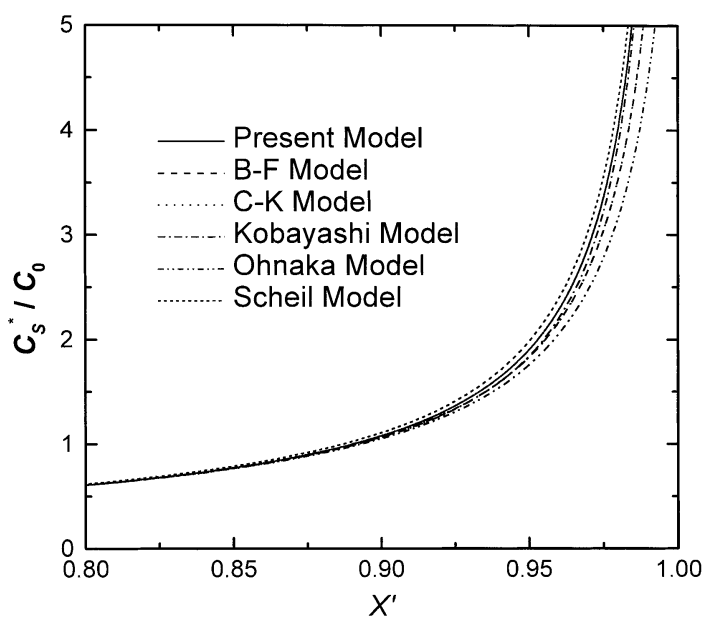

(b)

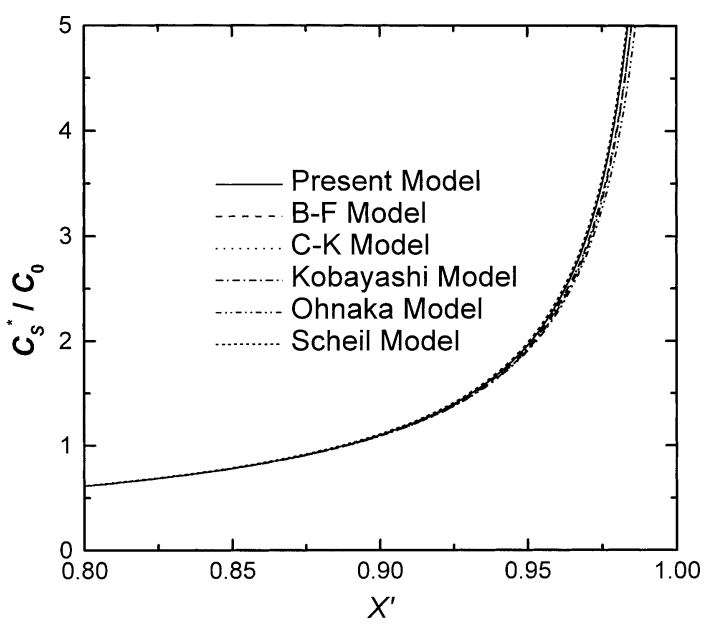

(c)

Fig. 3. Dimensionless solute concentration of solid at the solid/liquid interface during solidification in the absence of coarsening $(n \rightarrow \infty)$ with $k=0.16$ and $m=2$, for various Fourier numbers: (a) $\alpha=0.237$, (b) $\alpha=0.0327$, and (c) $\alpha=0.00996$.

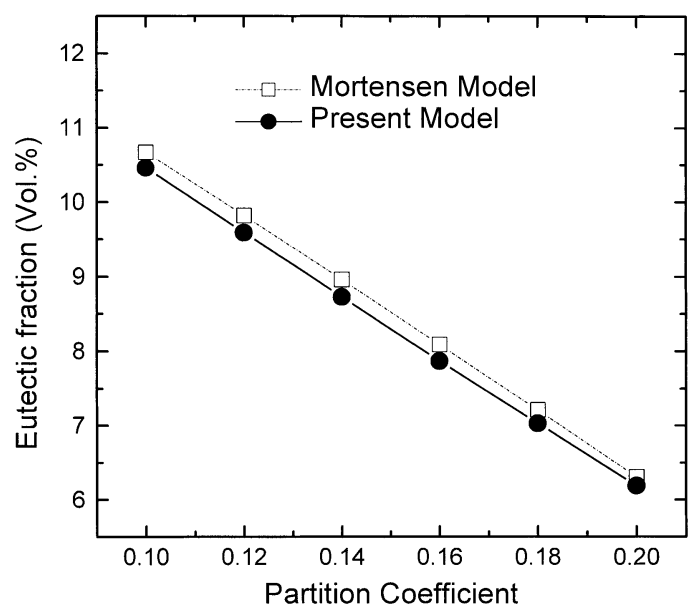

Fig. 4. Prediction of eutectic fractions in the absence of backdiffusion $(\alpha=0)$ with $k=0.16$ and $n=3$. Parabolic growth was assumed in the present model, and a constant cooling condition was assumed in the Mortensen model.

fore considered that the present model, in which the parameter $m$ accounts for the solidification condition, is more useful for practical application than the Mortensen model.

Figure 4 indicates the predicted eutectic fraction $\left(f_{\text {eut }}\right)$ both by the present model and Mortensen model. The amounts of eutectic fraction were predicted as a function of partition coefficient using the following equations.

$$
f_{\text {eut }}=1-\left.f_{\mathrm{S}}\right|_{C_{\mathrm{L}}=C_{\text {eut }}}
$$

where $C_{\text {eut }}$ indicates the eutectic composition. In Fig. 4, there is a little discrepancy between the two calculation results. This discrepancy is caused by the fact that the parabolic growth is assumed in the present model, but in the Mortensen model a constant cooling rate is assumed.

\subsection{Coarsening with Back-diffusion}

In order to verify the present model, the prediction of the fraction eutectic was compared with experimental results reported by Sarreal and Abbaschian ${ }^{10)}$ on directional solidification of an $\mathrm{Al}-4.9 \mathrm{wt} \% \mathrm{Cu}$ alloy. The conditions used in their experiments and the results are shown in Table 3. Thermal and physical properties used in the calculation are given in Table 4. In the present calculation, the reciprocal coarsening exponent was assumed to be 3 , which is a theoretical value, ${ }^{7,8)}$ and the reciprocal growth exponent was assumed to be 2 , which is the value for parabolic growth. ${ }^{8)}$

Figure 5 compares the measured eutectic fractions as a function of cooling rate with the predicted ones, made by various types of previous microsegregation models. As described in Table 2, the Mortensen model includes the effect of coarsening with no back-diffusion. Both effects of backdiffusion and coarsening are not considered in the Scheil's

Table 3. Experimental conditions and results for an $\mathrm{Al}-4.9 \mathrm{wt} \% \mathrm{Cu}$ alloy. ${ }^{10}$

\begin{tabular}{|c|c|c|c|c|c|}
\hline & $\begin{array}{c}\text { Cooling Rate } \\
(\mathrm{K} / \mathrm{s})\end{array}$ & $\begin{array}{c}\text { Solidification Time, } \\
t_{f}(\mathrm{~s})\end{array}$ & $\begin{array}{c}\text { Measured arm spacing, } \\
\lambda_{2}(\mu \mathrm{m})\end{array}$ & $\begin{array}{c}\text { Fourier number } \\
\alpha\end{array}$ & $\begin{array}{c}\text { Measured eutectic } \\
\text { fraction, } f_{\text {cuu }}(\text { Vol.\%) }\end{array}$ \\
\hline 1 & 0.1 & 980 & 90.86 & 0.237 & 5.54 \\
\hline 2 & 1.05 & 93.3 & 45.95 & 0.0884 & 6.52 \\
\hline 3 & 11.25 & 8.72 & 23.10 & 0.0327 & 6.84 \\
\hline 4 & 65 & 1.51 & 13.89 & 0.0157 & 7.16 \\
\hline 5 & 187 & 0.52 & 10.22 & 0.00996 & 7.50 \\
\hline 6 & 1700 & 0.058 & 5.39 & 0.00399 & 6.19 \\
\hline
\end{tabular}


Table 4. Thermal and physical properties of an $\mathrm{Al}-4.9 \mathrm{wt} \% \mathrm{Cu}$ alloy. ${ }^{8)}$

\begin{tabular}{ccl}
\hline Properties & \multicolumn{1}{c}{ Value } \\
\hline Mass diffusivity & $D_{S}\left(\mathrm{~m}^{2} / \mathrm{sec}\right)$ & $5.0 \times 10^{-13}$ \\
Partition coefficient & $k$ & 0.16 \\
Eutectic composition & $C_{\text {eut }}(\mathrm{wt} . \%)$ & 33.2 \\
\hline
\end{tabular}

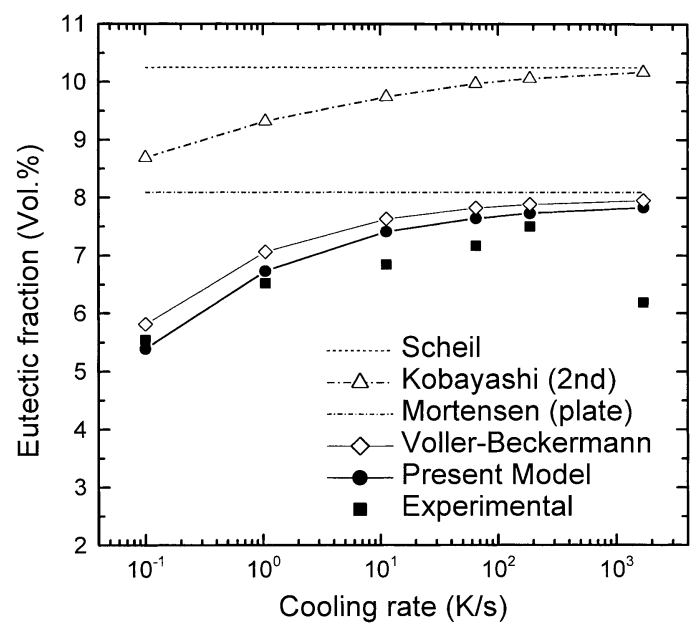

Fig. 5. Comparison of the predicted eutectic fractions for different microsegregation models with various cooling rates in directional solidification of $\mathrm{Al}-4.9 \mathrm{wt} \% \mathrm{Cu}$.

model. It is therefore reasonable to compare the two models for estimating the effect of coarsening. The predicted eutectic fraction by the Mortensen model is smaller than that by the Scheil's model, which is caused by the dilution effect of coarsening. Because of the same reason, the eutectic fractions predicted by the Voller-Beckerman (V-B) model ${ }^{9)}$ and the present model are smaller than those by the Kobayashi model in which the effect of back-diffusion alone is considered with no coarsening.

Similarly, let us compare the Kobayashi model with the Scheil's model in order to estimate the effect of back-diffusion. As shown in Fig. 5, the predicted eutectic fraction by the Kobayashi model is smaller than that by the Scheil's model. The difference of predicted eutectic fractions between the two models is caused by the dilution effect of back-diffusion. As the cooling rate increases, the dilution effect of back-diffusion decreases. In a similar reason, the dilution effect of back-diffusion is revealed by the difference between the Mortensen model and the present model. The V-B model shows a similar tendency. The effect of cooling rate on the dilution of back-diffusion can be explained by the Fourier number $(\alpha)$ since $\alpha$ is inversely proportional to the cooling rate as shown in Table 3. Thus, if the cooling rate is sufficiently large, both the $\mathrm{V}-\mathrm{B}$ model and the present model approach to the Mortensen model. It is also found that the present model shows a better agreement with the experimental results than the V-B model. This is caused by the fact that the effect of coarsening is directly included in the present model, but indirectly considered with the correction of the Fourier number in the V-B model. In addition, in case of the high cooling rate, $1700 \mathrm{~K} / \mathrm{s}$, the measured eutectic fraction is much smaller than the predicted ones. This discrepancy can be easily un- derstood from the fact that the effective partition coefficient under higher cooling rates is relatively larger than the equilibrium partition coefficient, which was used for predicting the amount of eutectic fraction. Nastac and Stefanescu reported that the effective partition coefficient of an $\mathrm{Al}-$ $4.9 \mathrm{wt} \% \mathrm{Cu}$ alloy increases rapidly as the cooling rate increases above $1 \mathrm{~K} / \mathrm{s} .{ }^{11)}$ It was also shown in Fig. 4 that the predicted eutectic fraction is inversely proportional to the partition coefficient.

\section{Conclusions}

An analytical model has been developed for predicting microsegregation in the secondary arm spacings of dendritic mushy regions. The present model was compared with other previous analytical models, and applied to the prediction of eutectic fractions in directional solidification of an $\mathrm{Al}-4.9 \mathrm{wt} \% \mathrm{Cu}$ alloy. The main results are summarized as follows:

(1) The present model includes both the dilution effects of back-diffusion and coarsening. The effect of back-diffusion was accounted for adopting the Ohnaka approximation for the concentration profile in solid with a modified parameter, and the effect of coarsening was directly accounted for by expanding the calculation domain during solidification.

(2) When the dilution effect of coarsening is not considered, the present model approaches to the Kobayashi model, whilst it approaches to the Mortensen model in the absence of back-diffusion.

(3) The predicted eutectic fractions by the present model are in good agreement with the measured ones. In addition, the present model shows a better agreement with the experimental results than other models.

(4) It is concluded that the present microsegregation model can be used for predicting the composition of microstructures and the fraction of eutectic or other phases in dendritic solidification of alloys.

\section{Acknowledgement}

This work was financially supported by POSCO (Pohang Iron and Steel Company) and by the Brain Korea 21 Project.

\section{Nomenclature}

$a \quad$ : Coefficient of the Ohnaka's approximation ${ }^{5)}$

C : Concentration

$D \quad$ : Mass diffusion coefficient

$f \quad$ : Fraction

$k \quad$ : Equilibrium partition coefficient

$L \quad$ : Length of the calculation domain

$m$ : Reciprocal growth exponent

$m_{\mathrm{L}} \quad$ : Liquidus slope

$n \quad$ : Reciprocal coarsening exponent

$R \quad$ : Cooling rate

$x \quad$ : Cartesian coordinates

$X$ : Position of the solidification front

$t \quad$ : Time

$\alpha \quad$ : Fourier number

$\beta \quad$ : Cooling rate $/\left(-m_{\mathrm{L}}\right)$

$\lambda_{2} \quad$ : Secondary dendrite arm spacing 


\section{Subscripts}

$0 \quad$ : Initial

f : Final

L : Liquid

$\mathrm{S} \quad$ : Solid

eut : Eutectic

Superscripts

* : Solidification front

, : Dimensionless

\section{REFERENCES}

1) T. Kraft and Y.A. Chang: J. Met., 49 (1997), 20.
2) M. C. Flemings: Solidification processing, McGraw-Hill, New York, (1977).

3) H. D. Brody and M. C. Flemings: Trans. Metall. Soc. AIME, 236 (1966), 615.

4) T. W. Clyne and W. Kurz: Metall. Trans. A, 12A (1981), 965.

5) I. Ohnaka: Trans. Iron Steel Inst. Jpn., 26 (1986), 1045.

6) S. Kobayashi: Trans. Iron Steel Inst. Jpn., 28 (1988), 728.

7) A. Mortensen: Metall. Trans. A, 20 (1989), 247.

8) V. R. Voller and C. Bechermann: Metall. Mater. Trans. A, 30A (1999), 2183.

9) V. R. Voller and C. Bechermann: Metall. Mater. Trans. A, 30A (1999), 3016.

10) J. A. Sarreal and G. J. Abbaschian: Metall. Trans. A, 17A (1986), 2063.

11) L. Nastac and D. M. Stefanescu: Metall. Trans. A, 24A (1993), 2107. 